\title{
Comments
}

\section{PsoReg - The Swedish Registry for Systemic Psoriasis Treatment}

\author{
The Registry's Design and Objectives \\ Marcus Schmitt-Egenolf \\ Department of Public Health and Clinical Medicine, Dermatology and Venereology, Umeå University, Umeå, Sweden
}

\section{Key Words}

Registries, psoriasis treatment $\cdot$ Psoriasis •

Safety, psoriasis treatment $\cdot$ Quality assurance, health

care $\cdot$ Ethics, medical $\cdot$ Drug therapy, psoriasis •

Pharmacogenetics - Clinical trials, shortcomings ·

Treatment outcome, psoriasis - Clinical competence,

tumor necrosis factor/therapeutic use •

Professional-patient relations

\begin{abstract}
With the introduction of new systemic drugs for the management of psoriasis, we felt an obligation in Sweden to establish a trusted tool to monitor their use. We formed PsoReg to create a solid, long-term database in order to analyze safety and effectiveness of different systemic psoriasis treatment regimens. PsoReg will provide information to help clinicians individualize therapy on a rational basis through evaluation of effectiveness and adverse effects in specific patient subgroups. Designed and managed by specialized health care professionals, PsoReg will enroll all psoriasis patients on systemic treatment to allow a fair comparison of old versus new-generation psoriasis treatments. PsoReg will even create benchmark data for quality assurance of the medical service. A web-based design allows real-time pharmacovigilance and enables the registry to assist clinicians in their day-to-day management of psoriasis patients. In this way PsoReg can become an integrated part of tomorrow's dermatology.

Copyright $\odot 2007$ S. Karger AG, Basel
\end{abstract}

\section{KARGER}

Fax +4161306 1234 E-Mail karger@karger.ch www.karger.com
(C) 2007 S. Karger AG, Base

$1018-8665 / 07 / 2142-0112 \$ 23.50 / 0$

Accessible online at:

www.karger.com/drm

\section{Introduction}

If our psoriasis patients ask us which therapy we suggest, they believe that we will suggest the optimal therapy for the individual patient according to the best of our knowledge. The question is: How good is our 'best knowledge'?

PsoReg is a tool to continuously improve this knowledge through analyses of the experience of all participating physicians. Because PsoReg is designed and managed by dermatologists, the entire process of creating, analyzing, and finally applying the collective knowledge to patient care is done within our profession, and by design protected from commercial influences.

The high prevalence of psoriasis in Sweden, combined with the advanced state-run epidemiological infrastructure, gives us a unique possibility to perform advanced analyses and to apply the results to the national health care process. PsoReg will provide a systematic but real-life picture of the diseased population in actual practice and can compensate for the inherent shortcomings of both randomized clinical trials and spontaneous reporting of side effects which have been discussed recently [1].

The main objectives of PsoReg are to assess the longterm safety and effectiveness of systemic psoriasis treatments and to identify target phenotypes that may allow for specific, individualized treatments. By establishing benchmarks of psoriasis care, PsoReg will also serve as a quality assurance tool. To allow for a fair analysis, the observed rate of adverse effects in patients receiving biologics will be examined alongside the rate in patients receiving conventional systemic treatment. 
The goal of PsoReg is to enable all physicians in Sweden who prescribe systemic psoriasis treatments to register and monitor their patients. However, to achieve our objectives with the shortest possible delay, a synthesis of results from registries across several countries is required. This synthesis becomes more efficient if the design of different registries is compatible. By publishing the PsoReg design in parallel to its launch, we want to stimulate the ongoing international foundation and collaboration of registries. In the light of the increased risk of infections and malignancies in rheumatoid arthritis patients treated with anti-tumor necrosis factor antibodies [2,3], the production of reliable data for psoriasis is essential. An overview of the PsoReg is given in the appendix. The different pages of the registry described there (Admission, Status, Treatment, Patient and Side effect report) are referred to in italics in the text.

\section{Design}

To allow uncomplicated access, PsoReg is web based and can offer both real-time pharmacovigilance and practical support to clinicians in their day-to-day management of psoriasis patients. It is one question for a physician to be convinced about the significance of a registry and another question to use it on a daily basis. Established registries need to fight against doctor burnout, where physicians who initially used a registry drop out later on due to time pressure. Therefore, to succeed, a register needs to provide benefits to the user: Time invested in the registration needs a pay back. At this point, PsoReg's Profile provides a structured electronic medical record for psoriasis care, and statistical, reporting, and search functionality will follow shortly.

\section{Safety}

If adverse drug reactions (ADRs) escape detection in clinical trials, there is a risk that they will even escape spontaneous reporting for a longer period of time, a lesson we learned from the cyclooxygenase- 2 inhibitors.

Conversely, as compared with the passive and unreliable system of spontaneous reporting, pharmacosurveillance with web-based registries is active and can recognize ADRs with the shortest possible delay. With PsoReg, we will be able to calculate the incidence rate of a given ADR, as we will know the number of affected individuals (the numerator) as well as the person-time at risk (the de- nominator). Moreover, we will have the chance to allocate ADRs to specific patient subpopulations or situations.

However, even a registry can fail to detect side effects, if the physician does not suspect an event to be related to the drug given. Consequently, in PsoReg pharmacovigilance is taken one step further, as the physician is also prompted on the Status page to update the record as any new concomitant conditions develop. In the safety context, this question works as a concomitant disease surveillance module. In this way, PsoReg can identify new ADRs, even if the individual clinician perceives no relationship between a medication and an adverse event. On the other hand, a drug may provide an unanticipated beneficial effect beyond the labeled indication. For example, in patients with rheumatoid arthritis, methotrexate was found to decrease the cardiovascular mortality [4]. The concomitant disease surveillance module allows us to detect such positive secondary drug effects too.

Paradoxically, the psoriasis phenotype can radically worsen under a therapy given, like the new onset of psoriatic arthritis in individuals treated for skin psoriasis [5]. PsoReg is designed to sense even those changes, as the questions concerning the psoriasis phenotype on the Status page here work as a module for phenotype surveillance.

\section{Effectiveness}

Given their limited duration, randomized clinical trials can hardly address the long-term effectiveness of systemic psoriasis therapies. Furthermore, randomized clinical trials are often designed by drug manufacturers to establish the efficacy in the most efficient manner; this typically involves selection of a homogeneous patient population that is not necessarily representative of real-life practice [6].

Decision makers in health care, however, need more valid data sources. PsoReg is built to objectively detect opportunities for improved disease management in real life by comparing the long-term effectiveness of different treatments and treatment combinations. To assure the best possible evaluation, the effectiveness of the therapy is evaluated by the development of the disease over time as assessed by both the physician on the Treatment page and the Patient himself. Active involvement of the patient in this step does not only result in an improved judgment of the effectiveness, it even emphasizes the role of the patient as a partner in the disease management process. By combining on the Patient side the more disease-specific Dermatology Life Quality Index (DLQI) [7] with a generic instrument to measure the quality of life, the 
EuroQol-5D [8], both cost-effectiveness and cost-utility calculations can be performed. Consequently, the disease burden of a psoriasis patient can be weighted against other diseases. In the future, those reliable data from the 'real life' may assure that our psoriasis patients have access to the best therapies available.

\section{Individualized Therapy}

Genetic evidence suggests that psoriasis is a heterogeneous disease [9]. This impression is corroborated by the dichotomy in therapy response between responders and nonresponders to a given therapy [10]. It might be possible that both risks and opportunities of different therapies can be attributed to patients with a specific profile. It is tempting to speculate how much more useful existing drugs could be, if we could predict a priori which patients are likely to respond optimally - and which patients have an elevated risk for adverse effects.

PsoReg collects considerable information on every registered patient in order to be able to search for particular patient profiles or constellations that are associated with good versus bad therapy responses and development of adverse events. As shown on the Status page (see appendix), special weight is placed on questions reflecting the known association of psoriasis with metabolic syndrome, smoking, and alcohol. For example, it is important to know whether patients suffering from obesity have a higher or lower risk of cardiovascular mortality under particular therapies.

\section{Pitfall: Confounding by Indication}

Biological therapies are quite new in the treatment of psoriasis. There is a clear need to compare effectiveness and safety of different biologics with each other and against conventional systemic treatments. However, even in a natural setting, this evaluation can produce misleading data, if not done carefully.

The likelihood for a given patient to receive a particular treatment depends on her/his psoriasis phenotype, comorbidities, and former treatments. However, these factors might be associated with adverse events in their own right. If a registry is not designed properly, it can consequently generate bias in the form of 'confounding by indication' [11]. This can be illustrated with the example of a patient who developed several nonmelanoma skin cancers after many years of both carcinogenic (PUVA) and immunosuppressant (cyclosporine) treatments. A physician might for that reason choose retinoids for further treatment. However, in an inadequately designed registry, the development of the next nonmelanoma skin cancer could mistakenly be computed as a result of the retinoid treatment. To be able to correct for such a bias, PsoReg asks several questions concerning earlier therapies and diseases in Admission and Status.

\section{Outlook}

A well-designed registry is a win-win situation for all involved parties. From a societal perspective, the registry works to avoid unnecessary suffering and costs. The participating physicians and patients are reassured to incorporate medical progress by using new drugs, as they know they are part of a pharmacovigilance and effectiveness testing network dedicated to individualized therapy.

\section{Appendix}

\section{Registration Flow}

The first page of the register, Admission, needs to be filled in on the first visit only. The four consecutive pages, Status, Treatment, Patient, and Lab values, are filled in on every consecutive visit. The Profile pops up after the registration is completed. The Side effect report can be used on any given point of time.

\section{Admission (fig. 1)}

This page documents the age of onset and its circumstances and former psoriasis treatments and former (not ongoing) morbidities of special interest, such as lymphoma, skin cancers, and severe infectious diseases.

\section{Status (fig. 2)}

This form registers the patient's general health data. The selection of questions reflects the known association of psoriasis with metabolic syndrome, smoking, and alcohol. As the extent of psoriasis in the family is prone to change, this question is asked here and not under Admission. The physician is prompted to continuously update the concomitant diseases and the psoriasis phenotype as well as the current nonpsoriasis treatment.

\section{Treatment (fig. 3)}

The psoriasis drug the patient currently receives is chosen from a pull-down menu. After completing the 
Fig. 1. Admission.

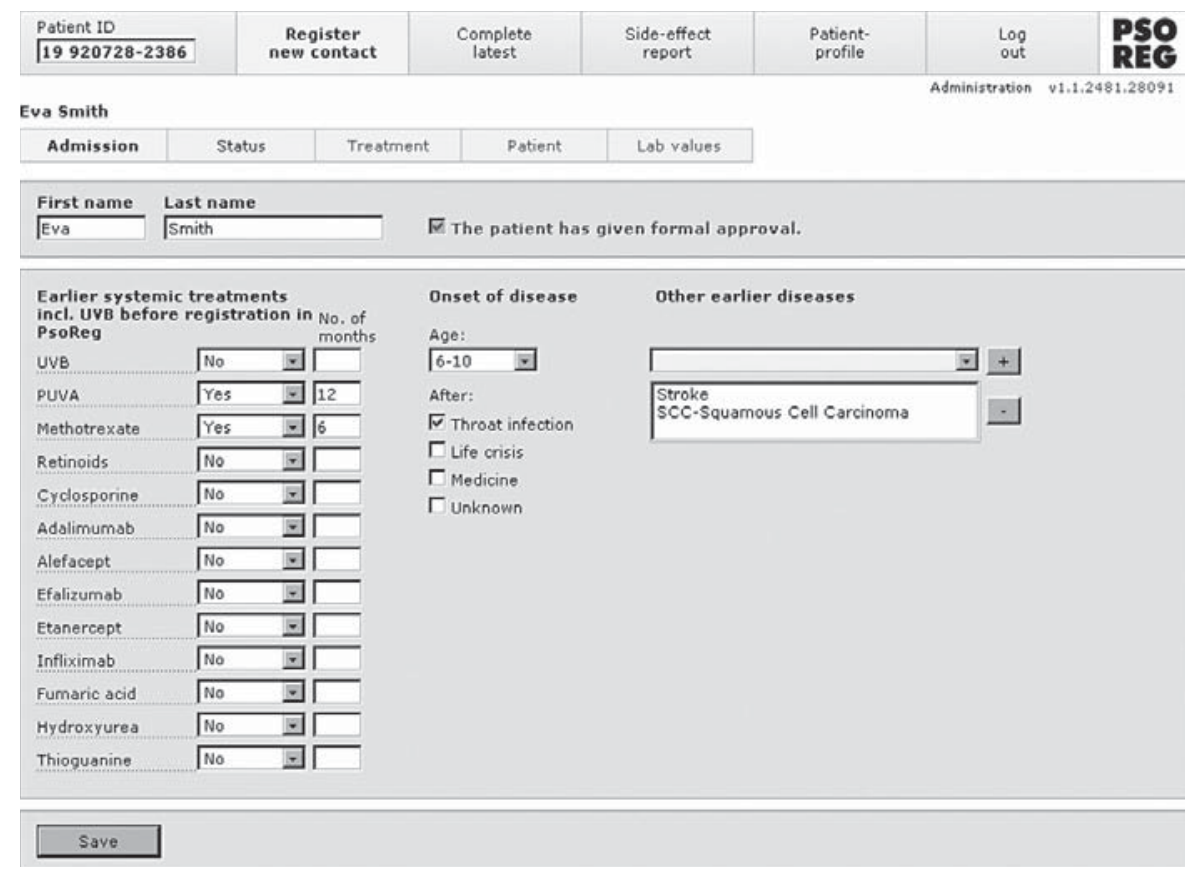

\begin{tabular}{l|l|c|c|c|c|c|}
\hline \begin{tabular}{l} 
Patient ID \\
\hline $\mathbf{1 9 9 2 0 7 2 8 - 2 3 8 6}$
\end{tabular} & $\begin{array}{c}\text { Register } \\
\text { new contact }\end{array}$ & $\begin{array}{c}\text { Complete } \\
\text { latest }\end{array}$ & $\begin{array}{c}\text { Side-effect } \\
\text { report }\end{array}$ & $\begin{array}{c}\text { Patient- } \\
\text { profile }\end{array}$ & $\begin{array}{c}\text { Log } \\
\text { out }\end{array}$ & $\begin{array}{l}\text { PSO } \\
\text { REG }\end{array}$ \\
\hline Eva Smith - new contact & & & Administration v1.1.2481.28091 \\
\hline
\end{tabular}

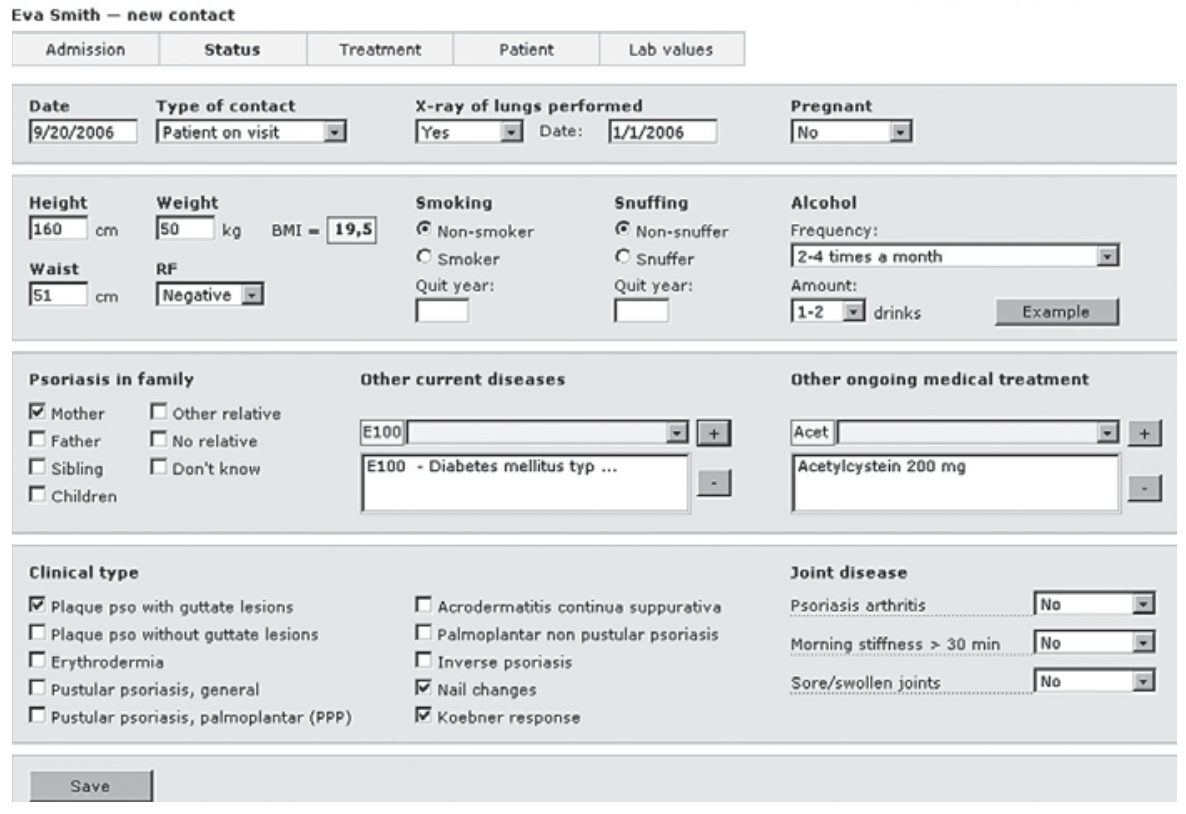

Fig. 2. Status.

dosage regimen, the treatment is displayed. In the case of combination therapies, all treatments can be displayed at once. The current disease activity, according to the physician, is registered by using both a visual analog scale (VAS) and the Psoriasis Activity and Severity Index (PASI).

The Swedish Registry for Systemic Psoriasis Treatment

\section{Patient (fig. 4)}

Here, the patient herself/himself is asked to quantify disease burden with the help of two VAS scales addressing disease activity in skin and joints. Additionally, a life quality instrument specific for skin diseases, the DLQI (Dermatology Life Quality Index), is employed alongside a generic instrument to measure the quality of life, the EuroQol-5D. 


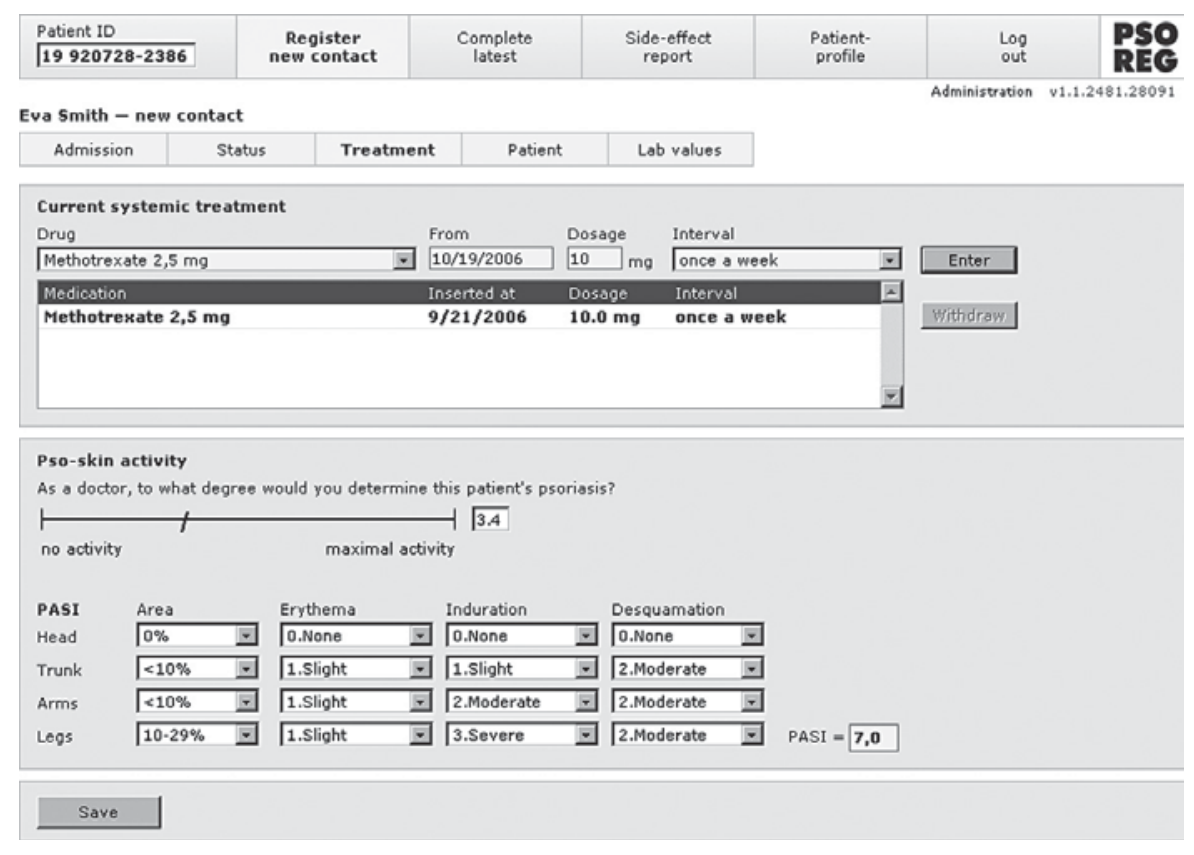

Fig. 3. Treatment.

(n)

\begin{tabular}{|l|c|c|c|c|c|c|}
\hline \begin{tabular}{|l|l|l|l|} 
Patient ID \\
\hline $\mathbf{1 9 9 2 0 7 2 8 - 2 3 8 6}$
\end{tabular} & $\begin{array}{c}\text { Register } \\
\text { new contact }\end{array}$ & $\begin{array}{c}\text { Complete } \\
\text { latest }\end{array}$ & $\begin{array}{c}\text { Side-effect } \\
\text { report }\end{array}$ & $\begin{array}{c}\text { Patient- } \\
\text { profile }\end{array}$ & $\begin{array}{c}\text { Log } \\
\text { out }\end{array}$ & $\begin{array}{l}\text { PSO } \\
\text { REG }\end{array}$ \\
\hline Eva Smith - new contact & & & Administration v1.1.2481.28091 \\
\hline
\end{tabular}

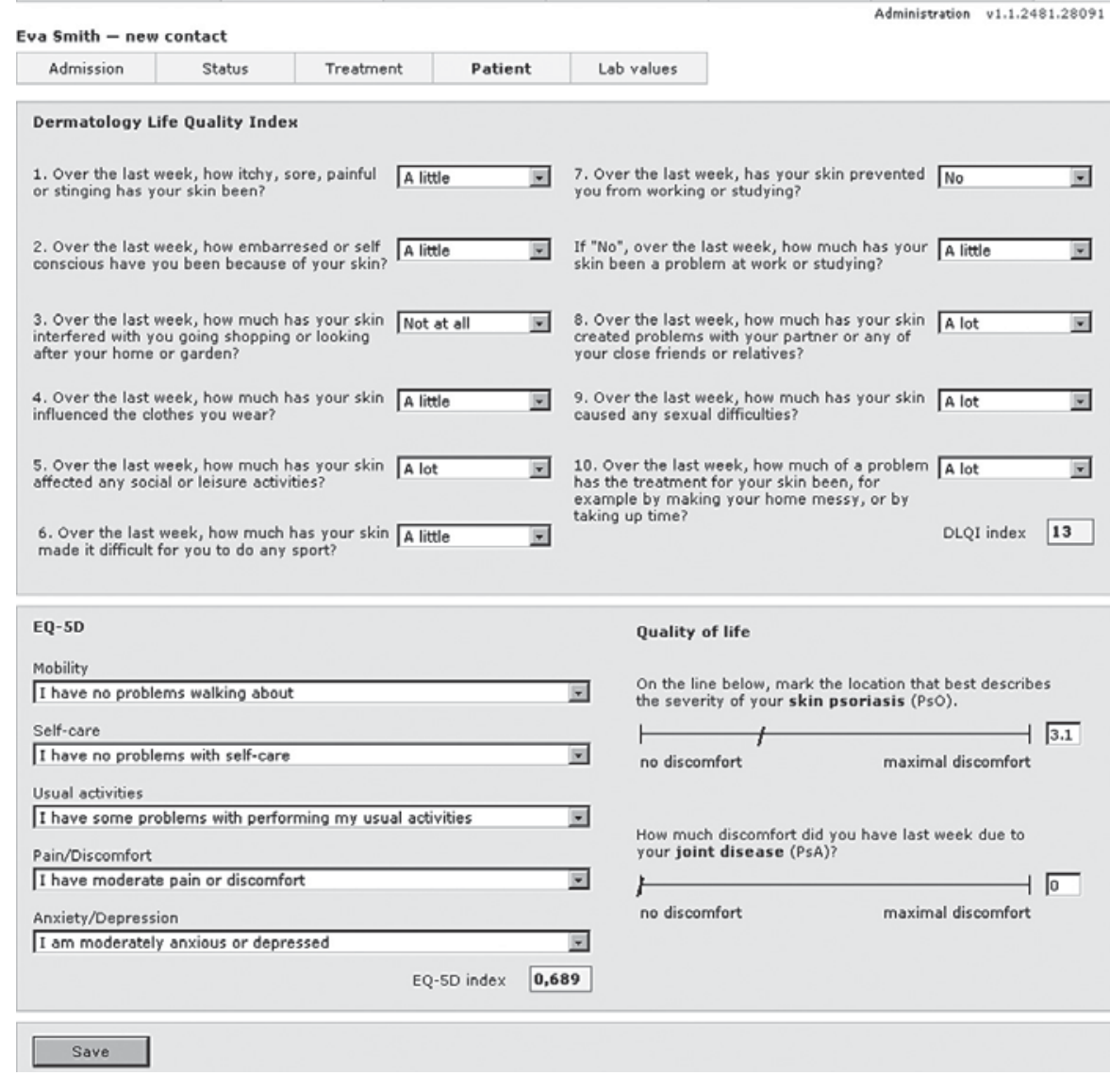

Fig. 4. Patient. 
Fig. 5. Side effect report.

\begin{tabular}{|c|c|c|c|c|c|c|}
\hline $\begin{array}{l}\text { Patient ID } \\
\qquad 19920728-2386\end{array}$ & $\begin{array}{l}\text { Register } \\
\text { new contact }\end{array}$ & $\begin{array}{c}\text { Complete } \\
\text { latest }\end{array}$ & $\begin{array}{l}\text { Side-effect } \\
\text { report }\end{array}$ & $\begin{array}{l}\text { Patient- } \\
\text { profile }\end{array}$ & $\begin{array}{l}\text { Log } \\
\text { out }\end{array}$ & $\begin{array}{l}\text { PSO } \\
\text { REG }\end{array}$ \\
\hline \multicolumn{7}{|c|}{ Administration v111.2481.28091 } \\
\hline \multicolumn{2}{|l|}{ Reaction or event } & Date of onset & Date ended & Ongoing? & & \\
\hline \multicolumn{2}{|c|}{ Description of reaction and event } & \multicolumn{3}{|c|}{ Seriousness } & & \\
\hline \multicolumn{2}{|l|}{ Suspected medication } & \multicolumn{2}{|l|}{ Action(s) taken } & & & \\
\hline & $\nabla$ & Drug withdrawn & $\nabla$ & & Append row & Delete \\
\hline Save & port & & & & & \\
\hline
\end{tabular}

Lab Values (not shown)

Lab values of special interest are registered here. In the future, this model will be completed from the referral management system/electronic patient record.

\section{Side Effect Report (fig. 5)}

The side effect report incorporated into the PsoReg can be forwarded digitally to the authorities. This is not only active quality assurance, but also a usability incentive which helps to integrate PsoReg into everyday care.

\section{Profile (not shown)}

The profile summarizes the medication along with the development of the disease over time. Individual patient contracts, such as smoking reduction, increased physical activities, and nutrition guidance, may be added. In addition, administrative information such as the time for the next consultation may be displayed. This profile can be printed and given to the patient at the end of the visit.

\section{Acknowledgments}

The author wants to express his gratitude to all those who helped to create PsoReg. Only few of them can be mentioned here, such as my Swedish colleagues from the PsoReg steering board: Ing-Marie Bergbrant, Göteborg; Ove Bäck, Lund; Berndt Stenberg, Umeå, and Mona Ståhle, Stockholm. Furthermore, the support of the following persons is also gratefully acknowledged: Linda Morfeldt and Gunilla Sjölin-Forsberg from the Swedish Medical Products Agency; Jonas Lekander from TietoEnator Healthcare, Sweden; Ulf Persson from the Swedish Institute for Health Economics; Daniel Mines from Wyeth Research, USA; my colleagues Knud Kragballe from Denmark and Tapio Rantanen from Finland, and Luigi Naldi from the Italian and Anthony Ormerod from the British Psoriasis Registry.

PsoReg is funded by Grants from public authorities: The Swedish Board of Health and Welfare and the Swedish Association of Local Authorities and Regions. However, without the financial support of our industry sponsors in Sweden, Abbott, Leo, Schering-Plough, Serono, and Wyeth, we would not have been able to realize PsoReg in an acceptable time frame.

\section{References}

1 Schmitt-Egenolf M: Psoriasis therapy in real life: the need for registries. Dermatology 2006;213:327-330.

-2 Bongartz T, Sutton AJ, Sweeting MJ, Buchan I, Matteson EL, Montori V: Anti-TNF antibody therapy in rheumatoid arthritis and the risk of serious infections and malignancies: systematic review and meta-analysis of rare harmful effects in randomized controlled trials. JAMA 2006;295:2275-2285.

-3 Listing J, Strangfeld A, Kary S, Rau R, von Hinueber U, Stoyanova-Scholz M, Gromnica-Ihle E, Antoni C, Herzer P, Kekow J, Schneider M, Zink A: Infections in patients with rheumatoid arthritis treated with biologic agents. Arthritis Rheum 2005;52: 3403-3412.
4 Choi HK, Hernan MA, Seeger JD, Robins JM, Wolfe F: Methotrexate and mortality in patients with rheumatoid arthritis: a prospective study. Lancet 2002;359:1173-1177.

5 Scheinfeld N: Efalizumab: A review of events reported during clinical trials and side effects. Expert Opin Drug Saf 2006;5:197-209.

-6 Davidoff F, DeAngelis CD, Drazen JM, Hoey J, Hojgaard L, Horton R, Kotzin S, Nicholls MG, Nylenna M, Overbeke AJ, Sox HC, Van Der Weyden MB, Wilkes MS: Sponsorship, authorship, and accountability. Lancet 2001; 358:854-856.

7 Finlay AY, Khan GK: Dermatology Life Quality Index (DLQI) - a simple practical measure for routine clinical use. Clin Exp Dermatol 1994;19:210-216.
-8 EuroQol Group: EuroQol - a new facility for the measurement of health-related quality of life. Health Policy 1990;16:199-208.

9 Online Mendelian Inheritance in Man OTJHU, Baltimore, Md., USA. MIM No.: $\{177900\}:\{4 / 14 / 2006\}$. World Wide Web URL: http://www.ncbi.nlm.nih.gov/omim/

10 Trepicchio WL, Ozawa M, Walters IB, Kikuchi T, Gilleaudeau P, Bliss JL, Schwertschlag U, Dorner AJ, Krueger JG: Interleukin-11 therapy selectively downregulates type I cytokine proinflammatory pathways in psoriasis lesions. J Clin Invest 1999;104:15271537.

11 Walker AM: Confounding by indication. Epidemiology 1996;7:335-336. 